\begin{tabular}{|c|l|}
\hline Title & Characteristic vector fields for first order partial differential equations \\
\hline Author(s) & Izumiya, S. \\
\hline Citation & Hokkaido University Preprint Series in Mathematics, 228, 1-9 \\
\hline Issue Date & 19942-1 \\
\hline DOI & 10.14943/83375 \\
\hline Doc URL & http://hdl.handle.net/2115/68979 \\
\hline Type & bulletin (article) \\
\hline File Information & pre228.pdf \\
\hline
\end{tabular}

Instructions for use 
CHARACTERISTIC VECTOR

FIELDS FOR FIRST ORDER

PARTIAL DIFFERENTIAL EQUATIONS

Shyuichi Izumiya

Series $\sharp 228$. February 1994 


\section{HOKKAIDO UNIVERSITY \\ PREPRINT SERIES IN MATHEMATICS}

$\sharp 198$ : T. Ozawa, Local decay estimates for Schrödinger operators with long range potentials, 17 pages. 1993.

$\sharp 199$ : A. Arai, N. Tominaga, Quantization of angle-variables, 31 pages. 1993.

$\sharp 200$ : S. Izumiya, Y. Kurokawa, Holonomic systems of Clairaut type, 17 pages. 1993.

\#. 201: K.-S. Saito, Y. Watatani, Subdiagonal algebras for subfactors, 7 pages. 1993.

\#202: K. Iwata, On Markov properties of Gaussian generalized random fields, 7 pages. 1993.

$\sharp 203$ : A. Arai, Characterization of anticommutativity of self-adjoint operators in connection with Clifford algebra and applications, 13 pages. 1993.

\#204: J. Wierzbicki, An estimation of the depth from an intermediate subfactor, 7 pages. 1993.

$\sharp 205$ : N. Honda, Vanishing theorem for the tempered distributions, 11 pages. 1993.

\#206: T. Hibi, Betti number sequences of simplicial complexes, Cohen-Macaulay types and Möbius functions of partially ordered sets, and related topics, 25 pages. 1993.

$\sharp 207$ : A. Inove, Regularly varying correlations, 23 pages. 1993.

$\sharp 208$ : S. Izumiya, B. Li, Overdetermined systems of first order partial differential equations with singular solution, 9 pages. 1993.

$\sharp 209$ : T. Hibi, Hochster's formula on Betti numbers and Buchsbaum complexes, 7 pages. 1993.

$\sharp 210$ : T. Hibi, Star-shaped complexes and Ehrhart polynomials, 5 pages. 1993.

$\sharp 211$ : $\quad$ S. Izumiya, G. T. Kossioris, Geometric singularities for solutions of single conservation laws, 28 pages. 1993.

\#212: A. Arai, On self-adjointness of Dirac operators in Boson-Fermion Fock spaces, 43 pages. 1993.

\#213: K. Sugano, Note on non-commutative local field, 3 pages. 1993.

$\sharp 214$ : A. Hoshiga, Blow-up of the radial solitions to the equations of vibrating membrane, 28 pages. 1993.

$\sharp 215$ : A. Arai, Scaling limit of anticommuting self-adjoint operators and nonrelativistic limit of Dirac operators, 35 pages. 1993.

$\sharp 216$ : Y. Giga, N. Mizoguchi, Existence of periodic solutions for equations of evolving curves, 45 pages. 1993.

$\sharp 217$ : T. Suwa, Indices holomorphic vector fields relative to invariant curves, 10 pages. 1993.

$\sharp 218$ : S. Izumiya, G. T. Kossioris, Realization theorems of geometric singularities for Hamilton-Jacobi equations, 14 pages. 1993.

$\sharp 219: \quad$ Y. Giga, K. Yama-uchi, On instability of evolving hypersurfaces, 14 pages. 1993.

$\sharp 220$ : W. Bruns, T. Hibi, Cohen-Macaulay partially ordered sets with pure resolutions, 11 pages. 1993.

\#221: S. Jimbo, Y. Morita, Ginzburg Landau equation and stable solutions in a rotational domain, 32 pages. 1993.

\# 222: T. Miyake, Y. Maeda, On a property of Fourier coefficients of cusp forms of half-integral weight, 12 pages. 1993.

$\sharp 223$ : I. Nakai, Notes on versal deformation of first order PDE and web structure, 34 pages. 1993.

$\sharp 224: \quad$ I. Tsuda, Can stoch astic renewal of maps be a model for cerebral cortex?, 30 pages. 1993.

$\sharp 225$ : H. Kubo, K. Kubota, Asymptotic behaviors of radial solutions to semilinear wave equations in odd space dimensions, 47 pages. 1994.

$\sharp 226$ : T. Nakazi, K. Takahashi, Two dimensional representations of uniform algebras, 7 pages. 1994.

$\sharp 227$ : N. Hayashi, T. Ozawa, Global, small radially symmetric solutions to nonlinear Schrödinger equations and a gauge transformation, 16 pages. 1994. 


\title{
CHARACTERISTIC VECTOR FIELDS FOR FIRST ORDER PARTIAL DIFFERENTIAL EQUATIONS
}

\author{
SHYUICHI IZUMIYA \\ Department of Mathematics, Faculty of Science, \\ Hokkaido University, Sapporo 060, JAPAN
}

\section{INTRODUCTION}

We consider the following two kinds of first order partial differential equations:

$$
\begin{array}{r}
\sum_{i=1}^{n} a_{i}(x, y) \frac{\partial y}{\partial x_{i}}-b(x, y)=0 \\
H\left(x_{1}, \ldots, x_{n}, \frac{\partial y}{\partial x_{1}}, \ldots, \frac{\partial y}{\partial x_{n}}\right)=0
\end{array}
$$

where $a_{i}(x, y), b(x, y)$ and $H(x, p)$ are $C^{\infty}$-functions. Here the equation (Q) is called a quasilinear first order partial differential equation (briefly, a quasilinear equation) and $(\mathrm{H})$ is called $a$ Hamilton-Jacobi equation. These equations are well studied in several articles $([2],[4],[5],[6],[8],[9],[10],[13],[14],[15],[18]$, etc. $)$. For the study of quasilinear equations, the theory of entropy solutions has provided the right weak setting (see, for example [9]). For Hamilton-Jacobi equations, the theory of viscosity solutions is appropriate one ([2],[3]). However, these notions of weak solutions have quite different features. Under the some assumptions, the entropy solutions are discontinuous and the viscosity solutions are continuous.

In this note we study the difference between quasilinear equations and HamiltonJacobi equations from the view point of the geometric theory of first order partial differential equations.

The goal of the present work is to give characterizations for each equation in terms of characteristic vector fields of equations. In relation to this study, Tsuji[16] considered what a Hamilton-Jacobi equation is. He has given an answer from the view point of the classical theory of first order partial differential equations (see also [17]). Here, we give an answer (Theorem 3.1 and Corollary 3.2) to Tsuji's question from the view point of the geometric theory. Moreover, we also give a characterization of quasilinear equations (Theorem 2.2).

We refer the following two typical examples of these equations.

Key words and phrases. Characteristic vector fileds, first odrder Partial Differential Equations. 
Example 0.1. We consider the following equations.

$$
\begin{aligned}
& \frac{\partial y}{\partial x_{1}}+2 y \frac{\partial y}{\partial x_{2}}=0 \\
& \frac{\partial y}{\partial x_{1}}+\left(\frac{\partial y}{\partial x_{2}}\right)^{2}=0 .
\end{aligned}
$$

We can explicitly solve these equations by the classical method of characteristics, when the initial condition is $y\left(0, x_{2}\right)=\sin x_{2}$. The pictures of the graph of geometric (multi-valued) solutions of these equations are given in Figure 1. These pictures are useful to understand the difference between these two equations. We can observe that the geometric solution for $\left(Q^{\prime}\right)$ is a smooth submanifold but for $\left(H^{\prime}\right)$ is not smooth in the $\left(x_{1}, x_{2}, y\right)$-space.

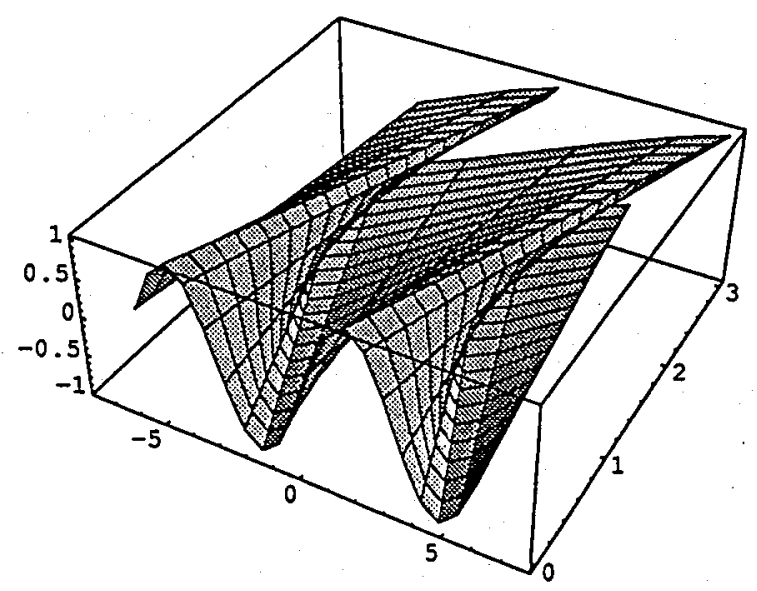

$Q^{\prime}$

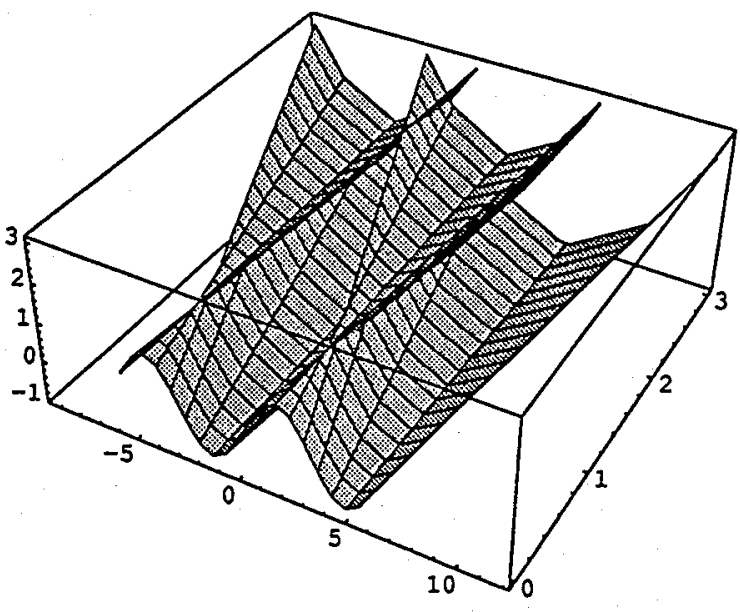

$\mathrm{H}^{\prime}$

Figure 1

All maps are differentiable of class $C^{\infty}$ unless stated otherwise.

\section{GeOMETRY OF First order partial DiffERENTIal EQUations}

We construct the geometric framework of equations in the projective cotangent bundle $\pi: P T^{*}\left(\mathbb{R}^{n} \times \mathbb{R}\right) \rightarrow \mathbb{R}^{n} \times \mathbb{R}$. We review geometric properties of this space. Consider the tangent bundle $\tau: T P T^{*}\left(\mathbb{R}^{n} \times \mathbb{R}\right) \rightarrow P T^{*}\left(\mathbb{R}^{n} \times \mathbb{R}\right)$ and the differential $\operatorname{map} d \pi: T P T^{*}\left(\mathbb{R}^{n} \times \mathbb{R}\right) \rightarrow T\left(\mathbb{R}^{n} \times \mathbb{R}\right)$ of $\pi$.

For any $X \in T P T^{*}\left(\mathbb{R}^{n} \times \mathbb{R}\right)$, there exists an element $\alpha \in T_{(x, y)}^{*}\left(\mathbb{R}^{n} \times \mathbb{R}\right)$ such that $\tau(X)=[\alpha]$. For an element $V \in T_{(x, y)}\left(\mathbb{R}^{n} \times \mathbb{R}\right)$, the property $\alpha(V)=0$ does not depend on the choice of representative of the class $[\alpha]$. Thus we can define the canonical contact structure on $P T^{*}\left(\mathbb{R}^{n} \times \mathbb{R}\right)$ by

$$
K=\left\{X \in T P T^{*}\left(\mathbb{R}^{n} \times \mathbb{R}\right) \mid \tau(X)(d \pi(X))=0\right\} .
$$

Because of the trivialization $P T^{*}\left(\mathbb{R}^{n} \times \mathbb{R}\right) \cong\left(\mathbb{R}^{n} \times \mathbb{R}\right) \times P\left(\mathbb{R}^{n} \times \mathbb{R}\right)^{*}$, we call $\left(\left(x_{1}, \ldots, x_{n}, y\right),\left[\xi_{1} ; \ldots ; \xi_{n} ; \eta\right]\right)$ a homogeneous coordinate, where $\left[\xi_{1} ; \ldots ; \xi_{n} ; \eta\right]$ is the homogeneous coordinate of the dual projective space $P\left(\mathbb{R}^{n} \times \mathbb{R}\right)^{*}$. 
It is easy to show that $X \in K_{((x, y),[\xi ; \eta])}$ if and only if $\sum_{i=1}^{n} \mu_{i} \xi_{i}+\lambda \eta=0$, where $d \tilde{\pi}(X)=\sum_{i=1}^{n} \mu_{i} \frac{\partial}{\partial x_{i}}+\lambda \frac{\partial}{\partial y}$.

We remark that $P T^{*}\left(\mathbb{R}^{n} \times \mathbb{R}\right)$ is a fibrewise compactification of the 1-jet space $J^{1}\left(\mathbb{R}^{n}, \mathbb{R}\right)$ as follows : We consider an open subset $U_{\eta}=\{((x, y),[\xi ; \eta]) \mid \eta \neq 0\}$ of $P T^{*}\left(\mathbb{R}^{n} \times \mathbb{R}\right)$. For any $((x, y),[\xi ; \eta]) \in U_{\eta}$, we have

$$
\left.\left.\left(x_{1}, \ldots, x_{n}, y\right),\left[\xi_{1} ; \ldots ; \xi_{n} ; \eta\right]\right)=\left(x_{1}, \ldots, x_{n}, y\right),\left[-\frac{\xi_{1}}{\eta} ; \cdots ;-\frac{\xi_{n}}{\eta} ;-1\right]\right)
$$

so that we may adopt the corresponding affine coordinate as

$$
\left(\left(x_{1}, \ldots, x_{n}, y\right),\left(p_{1} \ldots, p_{n}\right)\right)
$$

where $p_{i}=-\frac{\xi_{i}}{\eta}$. On $U_{\eta}$ we can easily show that $\theta^{-1}(0)=K \mid U_{\eta}$, where $\theta=$ $d y-\sum_{i=1}^{n} p_{i} d x_{i}$. This means that $U_{\eta}$ may be identified to the 1 -jet space $J^{1}\left(\mathbb{R}^{n}, \mathbb{R}\right)$. We call the above coordinate a canonical coordinate. Throughout the reminder of this paper, we use this identification so that we have

$$
J^{1}\left(\mathbb{R}^{n}, \mathbb{R}\right) \subset P T^{*}\left(\mathbb{R}^{n} \times \mathbb{R}\right)
$$

A first order partial differential equation (briefly, an equation) is defined to be a (not necessary closed) hypersurface $E \subset P T^{*}\left(\mathbb{R}^{n} \times \mathbb{R}\right)$.

By the philosophy of Lie, we may define the notion of solutions as follows: An immersion $i: L \rightarrow P T^{*}\left(\mathbb{R}^{n} \times \mathbb{R}\right)$ is said to be a Legendrian immersion if $\operatorname{dim} L=n$ and $d i_{q}\left(T_{q} L\right) \subset K_{i(q)}$ for any $q \in L$. A geometric solution of $E$ is a Legendrian immersion $i: L \rightarrow P T^{*}\left(\mathbb{R}^{n} \times \mathbb{R}\right)$ with Image $L \subset E$.

We now review the classical method of characteristics to solve equations (cf., [1],[13]). For a submanifold $N \subset P T^{*}\left(\mathbb{R}^{n} \times \mathbb{R}\right)$, a point $z \in N$ is said to be contact regular (or noncharacteristic) if $T N$ and $K$ transversally intersect at $z$. The characteristic direction at a contact regular point $z$ in an equation $E$ is the skeworthogonal complement of $T_{z} E \cap K_{z}$ in $K_{z}$, where the skew scalar product is defined as $\left.d \alpha\right|_{\alpha=0}$ and $\alpha$ is a local 1 -form such that $\alpha^{-1}(0)=K$. The Cauchy problem for an equation $E$ with initial submanifold $N$ consists of determining an integral manifold $L$ of the contact plane filed $K$, lying in $E$ and containing the initial submanifold $N$, where $N$ is an integral submanifold of $K$ with $\operatorname{dim}_{\mathbb{R}} N=n-1$. Then we have the following classical existence theorem of geometric solutions (cf., [1],[13]).

Theorem 1.1. Let $z$ be a contact regular point of the initial manifold $N$. Then there exists a neighbourhood $U$ of $z$ such that the geometric solution of the Cauchy problem for $E \cap U$ with initial condition $N \cap U$ exists and is locally unique.

We do not give the proof of the above theorem, however we remark that the characteristic direction of the equation at each point $z \in L$ is tangent to $L$. The integral curves of the field of characteristic directions on a contact regular equation $E$ are called the characteristics of the equation $E$, so that the characteristics through the initial manifold $N$ is contained in the geometric solution $L$. 
If the equation $E$ is written in the form $E \cap J^{1}\left(\mathbb{R}^{n}, \mathbb{R}\right)=F^{-1}(0)$, where $F$ : $J^{1}\left(\mathbb{R}^{n}, \mathbb{R}\right) \rightarrow \mathbb{R}$ is a submersion, then we can calculate explicitly the differential equation of the characteristics which is given by the following vector field on $J^{1}\left(\mathbb{R}^{n}, \mathbb{R}\right)$ under the canonical coordinate (cf., [13]):

$$
X_{F}=\sum_{i=1}^{n} \frac{\partial F}{\partial p_{i}} \frac{\partial}{\partial x_{i}}+\sum_{i=1}^{n}\left(p_{i} \frac{\partial F}{\partial p_{i}}-F\right) \frac{\partial}{\partial y}-\sum_{i=1}^{n}\left(\frac{\partial F}{\partial x_{i}}+p_{i} \frac{\partial F}{\partial y}\right) \frac{\partial}{\partial p_{i}} .
$$

In the last part of this section we refer the following fundamental property of the Legendrian submanifold in $J^{1}\left(\mathbb{R}^{n}, \mathbb{R}\right)$.

Fact 1.2. Let $L \subset J^{1}\left(\mathbb{R}^{n}, \mathbb{R}\right)$ be a Legendrian submanifold. Then $\operatorname{rank} d(\pi \mid L)_{q}<n$ if and only if $\operatorname{rank} d(\tilde{\pi} \mid L)_{q}<n$ for a point $q \in L$.

Here, $\pi: J^{1}\left(\mathbb{R}^{n}, \mathbb{R}\right) \rightarrow \mathbb{R}^{n} \times \mathbb{R}$ is given by $\pi(x, y, p)=(x, y)$ and $\tilde{\pi}: J^{1}\left(\mathbb{R}^{n}, \mathbb{R}\right) \rightarrow$ $\mathbb{R}^{n}$ is $\tilde{\pi}(x, y, p)=x$.

The proof is an easy exercise in the course of basic linear algebra, so that we omit it. However, this fact suggests that the geometric framework for quasilinear equation cannot be formulated in $J^{1}\left(\mathbb{R}^{n}, \mathbb{R}\right.$ ) (cf., Example 0.1).

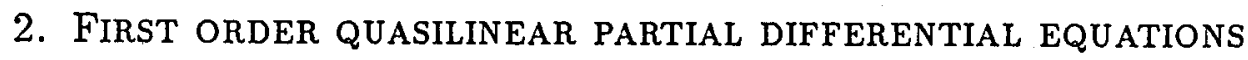

In this section we treat the quasilinear equation from the view point of the geometric theory which is introduced in the previous section. A quasilinear equation is defined to be a hypersurface

$$
E\left(a_{1}, \ldots, a_{n}, b\right)=\left\{((x, y),[\xi ; \eta]) \in P T^{*}\left(\mathbb{R}^{n} \times \mathbb{R}\right) \mid \sum_{i=1}^{n} a_{i}(x, y) \xi_{i}+b(x, y) \eta=0\right\}
$$

where $a_{i}(x, y), b(x, y)$ are $C^{\infty}$-function with $\left(a_{1}(x, y), \ldots, a_{n}(x, y)\right) \neq(0, \ldots, 0)$.

We remark that $\sum_{i=1}^{n} a_{i}(x, y) \xi+b(x, y) \eta=0$ if and only if $\sum_{i=1}^{n} a_{i}(x, y) p_{i}-$ $b(x, y)=0$ under the canonical coordinate of $U_{\eta}=J^{1}\left(\mathbb{R}^{n}, \mathbb{R}\right)$.

We consider the meaning of the notion of geometric solutions. Let $S$ be a smooth hypersurface in $\mathbb{R}^{n} \times \mathbb{R}$, then we have a unique Legendrian submanifold $\hat{S}$ in $P T^{*}\left(\mathbb{R}^{n} \times \mathbb{R}\right)$ such that $\pi(\hat{S})=S$ which is given as follows :

$\hat{S}=\left\{((x, y),[\xi ; \eta]) \mid\right.$ the vector $\sum_{i=1}^{n} \xi_{i} \frac{\partial}{\partial x_{i}}+\eta \frac{\partial}{\partial y}$ is a normal vector of $S$ in $\left.\mathbb{R}^{n} \times \mathbb{R}\right\}$.

It follows that if $L$ is a geometric solution of $E\left(a_{1}, \ldots, a_{n}, b\right)$ and $\pi \mid L: L \rightarrow \mathbb{R}^{n} \times \mathbb{R}$ is immersion, then we have $L=\widehat{\pi(L)}$.

We consider the condition of a smooth hypersurface $S$ that $\hat{S}$ is contained in $E\left(a_{1}, \ldots, a_{n}, b\right)$. For any $\left(x_{0}, y_{0}\right) \in S$, there exists a smooth submersion germ $f$ : $\left(\mathbb{R}^{n} \times \mathbb{R},\left(x_{0}, y_{0}\right)\right) \rightarrow(\mathbb{R}, 0)$ such that $\left(f^{-1}(0),\left(x_{0}, y_{0}\right)\right)=\left(S,\left(x_{0}, y_{0}\right)\right)$. A vector $\sum_{i=1}^{n} \mu_{i} \frac{\partial}{\partial x_{i}}+\lambda \frac{\partial}{\partial y}$ is tangent to $S$ at $(x, y) \in\left(S,\left(x_{0}, y_{0}\right)\right)$ if and only if it satisfies $\sum_{i=1}^{n} \mu_{i} \frac{\partial f}{\partial x_{i}}+\lambda \frac{\partial f}{\partial y}=0$ at $(x, y)$. Then we have the following representation of $\hat{S}$ :

$$
\left(\hat{S},\left(\left(x_{0}, y_{0}\right) ;\left[\xi_{0} ; \eta_{0}\right]\right)\right)=\left\{\left((x, y),\left[\frac{\partial f}{\partial x} ; \frac{\partial f}{\partial y}\right]\right) \mid(x, y) \in\left(S,\left(x_{0}, y_{0}\right)\right)\right\}
$$


We now consider the following vector field on $\mathbb{R}^{n} \times \mathbb{R}$ associated with the quasilinear equation $E\left(a_{1}, \ldots, a_{n}, b\right)$ :

$$
X\left(a_{1}, \ldots, a_{n}, b\right)=\sum_{i=1}^{n} a_{i}(x, y) \frac{\partial}{\partial x_{i}}+b(x, y) \frac{\partial}{\partial y} .
$$

Then we have the following proposition.

Proposition 2.1. Let $S$ be a smooth hypersurface in $\mathbb{R}^{n} \times \mathbb{R}$. Then $\hat{S}$ is a geometric solution of $E\left(a_{1}, \ldots, a_{n}, b\right)$ if and only if the vector field $X\left(a_{1}, \ldots, a_{n}\right.$, b) is tangent to $S$.

Proof. We have a local representation

$$
\left(\hat{S},\left(\left(x_{0}, y_{0}\right) ;\left[\xi_{0} ; \eta_{0}\right]\right)\right)=\left\{\left((x, y),\left[\frac{\partial f}{\partial x} ; \frac{\partial f}{\partial y}\right]\right) \mid(x, y) \in\left(S,\left(x_{0}, y_{0}\right)\right)\right\}
$$

where $f:\left(\mathbb{R}^{n} \times \mathbb{R},\left(x_{0}, y_{0}\right)\right) \rightarrow(\mathbb{R}, 0)$ is a submersion germ with $f^{-1}(0)=S$. It follows that $\hat{S} \subset E\left(a_{1}, \ldots, a_{n}, b\right)$ if and only if

$$
\sum_{i=1}^{n} a_{i}(x, y) \frac{\partial f}{\partial x_{i}}+b(x, y) \frac{\partial f}{\partial y}=0
$$

for $(x, y) \in\left(S,\left(x_{0}, y_{0}\right)\right)$. The last condition is equivalent to the condition that $X\left(a_{1}, \ldots, a_{n}, b\right)$ is tangent to $S$ at $(x, y)$.

In the classical theory, the vector field $X\left(a_{1}, \ldots, a_{n}, b\right)$ is called a characteristic vector field of the quasilinear equation $E\left(a_{1}, \ldots, a_{n}, b\right)$ (cf., [1]). However, we already define the notion of characteristic vector fields for general first order partial differential equations in $\S 1$. We study the relation between these notions of characteristic vector fields. On $J^{1}\left(\mathbb{R}^{n}, \mathbb{R}\right)$, we use the equation $F(x, y, p)=$ $\sum_{i=1}^{n} a_{i}(x, y) p_{i}-b(x, y)=0$. By definition, the characteristic vector field is given as follows :

$$
\begin{aligned}
X_{F} & =\sum_{i=1}^{n} a_{i}(x, y) \frac{\partial}{\partial x_{i}}+b(x, y) \frac{\partial}{\partial y} \\
& -\sum_{i=1}^{n}\left(\sum_{j=1}^{n}\left(\frac{\partial a_{i}(x, y)}{\partial x_{j}} p_{j}-\frac{\partial b(x, y)}{\partial x_{j}}\right)+p_{i}\left(\sum_{j=1}^{n} \frac{\partial a_{i}(x, y)}{\partial y} p_{j}-\frac{\partial b(x, y)}{\partial y}\right)\right) \frac{\partial}{\partial p_{i}}
\end{aligned}
$$

It follows that the projection of the characteristic vector field $d \pi\left(X_{F}\right)$ is equal to the vector field $X\left(a_{1}, \ldots, a_{n}, b\right)$. This fact characterizes the quasilinear equation as the following theorem shows.

Theorem 2.2. Let $E \subset P T^{*}\left(\mathbb{R}^{n} \times \mathbb{R}\right)$ be a closed equation. Then the following are equivalent.

(1) $E$ is a quasilinear equation. 
(2) There exists a function $F: J^{1}\left(\mathbb{R}^{n}, \mathbb{R}\right) \rightarrow \mathbb{R}$ with $\left(\frac{\partial F}{\partial p_{1}}, \ldots, \frac{\partial F}{\partial p_{n}}\right) \neq(0, \ldots, 0)$ on $F^{-1}(0)$ and $E \cap J^{1}\left(\mathbb{R}^{n}, \mathbb{R}\right)=F^{-1}(0)$ such that $d \pi\left(X_{F}\right)$ is a vector field on $\mathbb{R}^{n} \times \mathbb{R}$.

Proof. Assume that $d \pi\left(X_{F}\right)$ is a vector field on $\mathbb{R}^{n} \times \mathbb{R}$. Since

$$
d \pi\left(X_{F}\right)=\sum_{i=1}^{n} \frac{\partial F}{\partial p_{i}} \frac{\partial}{\partial x_{i}}+\sum_{i=1}^{n}\left(p_{i} \frac{\partial F}{\partial p_{i}}-F\right) \frac{\partial}{\partial y}
$$

the assumption means that $\frac{\partial F}{\partial p_{i}}(x, y, p)$ and $\sum_{i=1}^{n}\left(p_{i} \frac{\partial F}{\partial p_{i}}-F\right)(x, y, p)$ are independent on $\left(p_{1}, \ldots, p_{n}\right)$-variables, so that we may put $a_{i}(x, y)=\frac{\partial F}{\partial p_{i}}(x, y, p)$ for $i=1, \ldots, n$. It follows that

$$
\begin{aligned}
F(x, y, p) & =F(x, y, 0)+\int_{0}^{1} \frac{d F(x, y, t p)}{d t} d t \\
& =F(x, y, 0)+\int_{0}^{1} \sum_{i=1}^{n} \frac{\partial F}{\partial p_{i}}(x, y, t p) p_{i} d t \\
& =F(x, y, 0)+\sum_{i=1}^{n} \int_{0}^{1} a_{i}(x, y) d t p_{i} \\
& =F(x, y, 0)+\sum_{i=1}^{n} a_{i}(x, y) p_{i} .
\end{aligned}
$$

If we put $b(x, y)=-F(x, y, 0)$, then the equation has the form $F(x, y, p)=$ $\sum_{i=1}^{n} a_{i}(x, y) p_{i}-b(x, y)$. Since $\left(\frac{\partial F}{\partial p_{1}}, \ldots, \frac{\partial F}{\partial p_{n}}\right) \neq(0, \ldots, 0),\left(a_{1}(x, y), \ldots, a_{n}(x, y)\right) \neq$ $(0, \ldots, 0)$.

Thus we have $\left.E_{(} a_{1}, \ldots, a_{n}, b\right)=\overline{F^{-1}(0)}=E$. The proof of the converse direction is already proved by the previous arguments.

\section{HAMILTON-JACOBI EQUATIONS}

In this section we study the Hamilton-Jacobi equations by the similar view point as the case for quasilinear equations. Let $H\left(x_{1}, \ldots, x_{n}, p_{1}, \ldots, p_{n}\right)=0$ be a Hamilton-Jacobi equation, then the characteristic vector field is given as follows :

$$
X_{H}=\sum_{i=1}^{n} \frac{\partial H}{\partial p_{i}} \frac{\partial}{\partial x_{i}}-\sum_{i=1}^{n} \frac{\partial H}{\partial x_{i}} \frac{\partial}{\partial p_{i}}
$$

so that it exists on the $(x, p)$-space (i.e., the cotangent bundle $\left.T^{*} \mathbb{R}^{n}\right)$. Since we have the canonical decomposition $J^{1}\left(\mathbb{R}^{n}, \mathbb{R}\right) \cong T^{*} \mathbb{R}^{n} \times \mathbb{R}$, we give the geometric definition of the Hamilton-Jacobi equation as follows : Let $E \subset J^{1}\left(\mathbb{R}^{n}, \mathbb{R}\right) \subset P T^{*}\left(\mathbb{R}^{n} \times \mathbb{R}\right)$ be an equation. We say that $E$ is a Hamilton-Jacobi equation if there exists a submersion $H: T^{*} \mathbb{R}^{n} \rightarrow \mathbb{R}$ such that $E=H^{-1}(0)$. We introduce another important class of equations. We say that $E$ is a graphlike equation if there exists a function $H: T^{*} \mathbb{R}^{n} \rightarrow \mathbb{R}$ such that $E=\left\{(x, y, p) \in J^{1}\left(\mathbb{R}^{n}, \mathbb{R}\right) \mid y=H(x, p)\right\}$. The notion of graphlike equations is introduced by Kossowski [11] in order to study singular 
solutions of equations. The most famous example is the Clairaut equation which is given by

$$
y=\sum_{i=1}^{n} x_{i} p_{i}+f\left(p_{1}, \ldots, p_{n}\right)
$$

It is well known that the Clairaut equation is completely integrable with singular solution and the singular solution is the envelope of the family of graphs of the complete solution. In [7] we have shown that the set of contact singular points of an equation $E$ is an $n$-dimensional submanifold if and only if the equation has a singular solution (in the strict sense) and the equation is (locally) graphlike near the singular solution.

We have the following characterization theorem.

Theorem 3.1. Let $E \subset J^{1}\left(\mathbb{R}^{n}, \mathbb{R}\right) \subset P T^{*}\left(\mathbb{R}^{n} \times \mathbb{R}\right)$ be an equation. Then the following are equivalent.

(1) $E$ is a Hamilton-Jacobi equation or a graphlike equation.

(2) There exists a submersion $F: J^{1}\left(\mathbb{R}^{n}, \mathbb{R}\right) \rightarrow \mathbb{R}$ such that $F^{-1}(0)=E$ and $d \Pi\left(X_{F}\right)$ is a vector field on $T^{*} \mathbb{R}^{n}$, where $d \Pi: J^{1}\left(\mathbb{R}^{n}, \mathbb{R}\right) \rightarrow T^{*} \mathbb{R}^{n}$ is the canonical projection.

Proof. Suppose that the condition (2) holds, then the characteristic vector field is given as follows:

$$
X_{F}=\sum_{i=1}^{n} \frac{\partial F}{\partial p_{i}} \frac{\partial}{\partial x_{i}}+\sum_{i=1}^{n}\left(p_{i} \frac{\partial F}{\partial p_{i}}-F\right) \frac{\partial}{\partial y}-\sum_{i=1}^{n}\left(\frac{\partial F}{\partial x_{i}}+p_{i} \frac{\partial F}{\partial y}\right) \frac{\partial}{\partial p_{i}}
$$

It follows that $d \Pi\left(X_{F}\right)$ is a vector field on $T^{*} \mathbb{R}^{n}$ if and only if $\frac{\partial F}{\partial p_{i}}(x, y, p)$ and $\frac{\partial F}{\partial x_{i}}(x, y, p)+p_{i} \frac{\partial F}{\partial y}(x, y, p)$ depends only on $(x, p)$-variables for $i=1, \ldots, n$.

We have

$$
\begin{aligned}
F(x, y, p) & =F(x, y, 0)+\int_{0}^{1} \frac{d F(x, y, t p)}{d t} d t \\
& =F(x, y, 0)+\int_{0}^{1} \sum_{i=1}^{n} \frac{\partial F}{\partial p_{i}}(x, y, t p) p_{i} d t \\
& =F(x, y, 0)+\sum_{i=1}^{n}\left(\int_{0}^{1} \frac{\partial F}{\partial p_{i}}(x, y, t p) d t\right) p_{i}
\end{aligned}
$$

Since $\frac{\partial F}{\partial p_{i}}(x, y, p)$ are independent on the $y$-variable, we may put

$$
F(x, y, 0)=a(x, y) \text { and } \sum_{i=1}^{n}\left(\int_{0}^{1} \frac{\partial F}{\partial p_{i}}(x, y, t p) d t\right) p_{i}=h(x, p) .
$$

Moreover, by the condition that $\frac{\partial F}{\partial x_{i}}(x, y, p)+p_{i} \frac{\partial F}{\partial y}(x, y, p)$ are independent on the $y$-variable,

$$
\frac{\partial a}{\partial x_{i}}(x, y)+\frac{\partial h}{\partial x_{i}}(x, p)+p_{i} \frac{\partial a}{\partial y}(x, y)
$$


are independent on the $y$-variable. It is equivalent to the following condition:

$$
\frac{\partial^{2} a}{\partial x_{i} \partial y}(x, y)+p_{i} \frac{\partial^{2} a}{\partial y^{2}}(x, y) \equiv 0
$$

for $i=1, \ldots, n$. Differentiate the above equalities with respect to $p_{i}$-variables, we have $\frac{\partial^{2} a}{\partial x_{i} \partial y}(x, y) \equiv 0$ and $\frac{\partial^{2} a}{\partial y^{2}}(x, y) \equiv 0$, so that $\frac{\partial a}{\partial x_{i}}(x, y)$ and $\frac{\partial a}{\partial y}(x, y)$ depend only on $\left(x_{1}, \ldots, x_{n}\right)$-variables for $i=1, \ldots, n$. Then we may put $\frac{\partial a}{\partial x_{i}}(x, y)=b_{i}(x)$ and $\frac{\partial a}{\partial y}(x, y)=c(x)$. Thus we have

$$
\begin{aligned}
a(x, y) & =a(0,0)+\int_{0}^{1} \frac{d a(t x, t y)}{d t} d t \\
& =a(0,0)+\int_{0}^{1}\left(\sum_{i=1}^{n} \frac{\partial a}{\partial x_{i}}(t x, t y) x_{i}+\frac{\partial a}{\partial y}(t x, t y)\right) d t \\
& \left.=a(0,0)+\sum_{i=1}^{n}\left(\int_{0}^{1} \frac{\partial a}{\partial x_{i}}(t x, t y) d t\right) x_{i}+\left(\int_{0}^{1} \frac{\partial a}{\partial y}(t x, t y)\right) d t\right) y \\
& =a(0,0)+\sum_{i=1}^{n}\left(\int_{0}^{1} b_{i}(t x) d t\right) x_{i}+\left(\int_{0}^{1} c(t x) d t\right) y \\
& =B(x)+C(x) y
\end{aligned}
$$

where we put $B(x)=a(0,0)+\sum_{i=1}^{n}\left(\int_{0}^{1} b_{i}(t x) d t\right) x_{i}$ and $C(x)=\int_{0}^{1} c(t x) d t$.

Since $\frac{\partial a}{\partial y}(x, y)=C(x)$, we have $\frac{\partial C}{\partial x_{i}}(x)=\frac{\partial^{2} a}{\partial y \partial x_{i}}(x, y)=\frac{\partial^{2} a}{\partial x_{i} \partial y}(x, y) \equiv 0$, so that $C(x)=c$ (constant). We put $H(x, p)=B(x)+h(x, p)$, then we have

$$
F(x, y, p)=c y+H(x, p) \text {. }
$$

If $c=0$, then the equation is a Hamilton-Jacobi equation. If $c \neq 0$, then we have

$$
F^{-1}(0)=\left\{(x, y, p) \mid y+\frac{1}{c} H(x, p)=0\right\}
$$

it is a graphlike equation. The proof for converse direction is given by direct calculations. This completes the proof.

We also have the following characterization theorem of Hamilton-Jacobi equations as a corollary of the above theorem.

Corollary 3.2. Under the same assumption as in Theorem 3.1, the following are equivalent.

(1) $E$ is a Hamilton-Jacobi equation.

(2) There exists a submersion $F: J^{1}\left(\mathbb{R}^{n}, \mathbb{R}\right) \rightarrow \mathbb{R}$ such that $F^{-1}(0)=E$ and the characteristic vector field $X_{F}$ is a vector field on $T^{*} \mathbb{R}^{n}$.

Proof. Since $X_{F}$ is a vector field on $T^{*} \mathbb{R}^{n}$, then $d \Pi\left(X_{F}\right)=X_{F}$, so that the equation is written in the form $F(x, y, p)=c y+H(x, p)$, by the proof of Theorem 3.1. If $c \neq 0$, then $X_{F}$ is not a vector field on $T^{*} \mathbb{R}^{n}$. The proof for converse direction is also given by direct calculations. 


\section{REFERENCES}

1. V. I. Arnol'd, Geometric Methods in the Theory of Ordinary Differential Equations, Springer-Verlag, 1983.

2. G. Crandall and P. -Lions, Viscosity solutions of Hamilton-Jacobi equations, Trans. Amer. Math. Soc. 277 (1983), 1-42.

3. M. G. Crandall, H. Ishii and P.-Lions, User's guide to viscosity solutions of second order partial differential equations, Bull. Amer. Math. Soc. 27 (1992), 1-67.

4. J. J. Duistermaat, Oscillatory integrals, Lagrange immersions and Unfolding of singularities, Commu. pure and applied Math. 27 (1974), 207-281.

5. J. Guckenheimer, Solving a single conservation law, Lecture notes in Mathematics 468, Springer Verlag, New York, 1975, pp. 108-134.

6. S. Izumiya, Perestroikas of optical wave fronts and graphlike Legendrian unfoldings, J. of Differential Geometry 38 (1993), 485-500.

7. S. Izumiya, Singular solutions of first-order differential equations, Bull. London Math. Soc. (to appear).

8. S. Izumiya and G. Kossioris, Semi-local classification of geometric singularities for HamiltonJacobi equations, J. Differential Equations (to appear).

9. S. Izumiya and G. Kossioris, Geometric singularities for solutions of single conservation laws, preprint.

10. G. Jennings, Piecewise smooth solutions of a single conservation law exist, Adv. in Math $\mathbf{3 3}$ (1979), 192-205.

11. M. Kossowski, first-order partial differential equations with singular solution, Indiana Univ. Math. Jour. 35 (1986), 209-223.

12. S. N. Kruzkov, First order quasilinear equations in several independent variables, Math. USSR Sb. $10(1970), 217-243$.

13. V. V. Lychagin, Local classification of non-linear first-order partial differential equations, Russian Math. Surveys 30 (1975), 105-175.

14. S. Nakane, Formation of shocks for a single conservation law, SIAM J. Math. Anal. 19 (1988), 1391-1408.

15. M. Tsuji, Formation of singularities for Hamilton-Jacobi equation II, J. Math. Kyoto Univ. 26 (1986), 299-308.

16. M. Tsuji, Prolongation of classical solutions and singularities of generalized solutions, Ann. Inst. Henri Poincaré (Analyse non linéaire) 7 (1990), 505-523.

17. M. Tsuji and T. T. Li, Remarks on Characteristics of Partial Differential Equation of First Order, Funkcialaj Ekvacioj 32 (1989), 157-162.

18. D. Wagner, The Riemann problem in two space dimensions for a single conservation law, SIAM J. Math. Ann. 14 (1983), 534-559. 